\title{
Complementary therapeutic approaches in asthma
}

\author{
MAIEREAN Anca ${ }^{1}$, CIUMARNEAN Lorena ${ }^{2}$, ALEXESCU Teodora Gabriela ${ }^{2}$, \\ DOMOKOS Bianca ${ }^{3}$, RAJNOVEANU Ruxandra ${ }^{3}$, ARGHIR Oana ${ }^{4}$, TODEA Doina ${ }^{3}$, \\ BUZOIANU Anca Dana ${ }^{5}$, DOGARU Gabriela ${ }^{6}$, BORDEA Roxana Ioana ${ }^{7}$
}

Corresponding author: MAIEREAN Anca, E-mail: ancalupascu91@gmail.com,

1. „Iuliu Hatieganu” University of Medicine and Pharmacy, Cluj Napoca, Romania

2. „Iuliu Hatieganu” University of Medicine and Pharmacy, Department of Internal Medicine, Cluj Napoca, Romania

3. "Iuliu Hatieganu" University of Medicine and Pharmacy, Department of Pneumology, Cluj Napoca, Romania

4. "Ovidius" University from Constanta, Romania

5. „Iuliu Hatieganu” University of Medicine and Pharmacy, Department of Pharmacology, Cluj Napoca, Romania

6. „Iuliu Hatieganu” University of Medicine and Pharmacy, Medical Rehabilitation Department, Cluj Napoca, Romania

7. „Iuliu Hatieganu” University of Medicine and Pharmacy, Dentistry Faculty, Cluj Napoca, Romania

\begin{abstract}
Asthma is defined by The Initiative for Asthma (GINA 2018) as a heterogeneous disease, which include chronic airway inflammation and a history of respiratory symptoms. In the last decades asthma had a rise in prevalence, becoming one of the most frequent diagnosed diseases in the world. The main goals of asthma management are to achieve good symptom control, minimize the risks of exacerbations, decrease rescue medication intake, improve the quality of life by decreasing respiratory system inflammation and ameliorating the patient's lung function. Beside effective medications, asthma continues to impair quality of life for most patients. Due to the difficulty of controlling symptoms and exacerbations, the need of developing complementary options of treatment is increasing in order to achieve an optimum control and a lower risk of acute episodes or fatal events. Pulmonary rehabilitation is suggested for asthma patients when adequate medical therapy poorly control the symptoms and mental, physical or social consequences of illness persist during the daily life. The following non-drug therapy components are included in the rehabilitation program: physical training, comprehensive smoking cessation program, comprehensive patient education, respiratory physiotherapy, psychosocial support and comprehensive nutritional counseling.

These complementary therapies have been proven to improve muscle strength, exercise capacity and symptomatology. Also, it has been associated to fewer exacerbations and a lower use of rescue medication, leading to a better quality of life.
\end{abstract}

Key words: asthma, quality of life, symptomatology, rehabilitation, physiotherapy,

\section{Introduction}

The Initiative for Asthma (GINA 2018) defines asthma as a heterogeneous disease, usually characterized by chronic airway inflammation and a history of respiratory symptoms that vary over time and in intensity, including, shortness of breath, wheezing, chest tightness and cough, together with variable expiratory airflow limitation $(1,2)$. To establish the asthma diagnosis is challenging, relying on the patients' symptoms and objective examination of lung function (3).

Asthma it is a condition that remains incurable and achieves a poor control. Even though there are many effective medications, new approaches and complementary therapies to improve asthma control are urgently required $(2,3)$.

\section{Prevalence}

Asthma has a rise in prevalence with an estimated number of 300 million individuals globally, becoming one of the most frequent diagnosed diseases in the world (2). Moreover, the prevalence of asthma has been increasing since the 1950s worldwide, and seems to be steadily increasing in parallel with the urbanization (4).

The prevalence of asthma varies between countries, with prevalence estimates of $15.3 \%$ in England, $14.1 \%$ in Canada, $10.9 \%$ in the United States of America, 6.9\% in Germany, 6.7\% in Japan and 4.5\% in Italy. Also, the asthma prevalence was higher among women than men $(4,5)$. Several studies showed that the atopy is strongly associated with asthma, especially in young adults, teenagers and children which may indicate an increase in asthma prevalence during the forthcoming years also in occidental areas (4).

The incidence of asthma in the world has reached worrying levels, especially in the developed countries where this disorder has reached epidemic proportions (6). 


\section{Etiology}

The etiology of asthma is not well established, but many risk factors have been identified, including gene-environment interactions, such as respiratory infections, especially viral infections in the early life, atopic conditions and inhalant allergens, vitamin D, chemical exposure, pollutants, tobacco smoke and stress. Genetic studies have found hundreds of genes involved, but genetics do not entirely explain asthma (ㄱ).

Strong connections occur between asthma and allergies. Felman et al. theorize that $75 \%$ of adults with ages between 20 and 40 years and $65 \%$ with ages over 55 years have allergies. Atopy plays key role in asthma evolution, atopic asthma being one of the most common types of asthma. Environmental allergens lead to overproduction of $\operatorname{IgE}$ antibodies and trigger asthmatic reactions. Atopy is a kind of allergic hypersensitivity that leads to allergic reactions by increasing the production of $\operatorname{IgE}$ antibodies which is responsible for eczema, nettle rush, eye conditions (allergic conjunctivitis) and hay fever (allergic rhinitis) $(8,9)$.

Taking into account the environmental factors, it is a well known fact that asthma symptoms often occur because of indoor and outdoor air pollution. Common sources of indoor allergens include animal proteins, mostly from cat and dog dander, dust mites, cockroaches, fungi, mold, household cleaners, paints and secondhand smoke. Also, outdoor allergens are pollen, ozone, pollution, radon, cold temperatures, nitric oxide (NO2). NO2 is a gas that is produced in the process of combustion, gas heat or by woodburning stoves in homes, so it is a result of traffic and affects both allergic and nonallergic asthmatic patients $(8,10,11)$. In addition, air pollution and climate change have a negative impact on human health, by making the airways susceptible to allergens, leading to airway congestion, extra secretions of mucus and constriction or even simulating attacks of asthma $(8,12)$.

Tobacco smoke in also linked to an increased risk of asthma, a higher risk of inspiratory infections due to excess of mucus, wheezing, and other respiratory conditions worsening the patients clinical status by symptoms like breathlessness and coughing $(8,13)$. The mechanism by which smoking induces inflammatory changes should be interpreted both by acute and chronic exposure to cigarette smoking, inflammation progression becoming more important as the exposure to smoking extends $(8,14)$.

Another factor incriminated in asthma development is stress. The control of asthma during stressful times is influenced by individual behavior including smoking or emotional response that might trigger asthma attacks (8).

\section{Patophysiology}

The pathophysiology in asthma includes several mechanisms, such as cellular inflammation and tissue remodeling. The most common inflammation in the lower airway is type 2 inflammation involving type 2 $\mathrm{T}$ helper cell lymphocyte (TH-2) and most likely arises from a combination of genetic predisposition, alterations in the microbiome and environmental exposures. Type 2 inflammation is likely seen in eosinophilic disorders, allergic diseases and parasite infections, predisposing to asthma and have a good response to corticosteroids leading to an easier management. Tissue remodeling is relied on pathological alterations that primarily occur in the submucosa and mucosa, including smooth muscle hypertrophy and hyperplasia, goblet cell hyperplasia, hypertrophy of submucosal mucus glands, subepithelial fibrosis and collagen deposition, increased blood vessels in the submucosa, inflammatory cell infiltrate and submucosal edema (7).

\section{Diagnosis}

The diagnostic of asthma is established on the basis of symptoms and evaluation of lung function. Asthma is defined by 2 components: (1) history of respiratory symptoms such as chest tightness, wheezing, shortness of breath and cough, symptoms that vary in intensity and severity over time and (2) airflow limitation or variable expiratory airway obstruction $(1,15)$.

\section{Clinical features}

In most cases, the inflammation underlying asthma is present, causing important symptomatology, but in some cases asthma is found without being accompanied by symptoms. Moreover, the control of inflammation is a key component in the management of asthma. The absence of symptoms can lead to poor self awareness of asthma and noncompliance to available treatments. The tissue remodeling and associated inflammation cause a plenitude of symptoms like breathlessness, chest tightness, wheezing, cough and exercise-induced symptoms (16). As mentioned before, some triggers, such as 
upper respiratory viral infections (usually rhinovirus) can increase the inflammation and can generate asthma attacks (7). Also, acute exacerbations are frequent in non-controlled asthma, so in these periods, symptoms like shortness of breath, wheezing, chest tightness and cough tend to be worse at night $(\underline{7}, 17)$.

Most of the episodes are resolved with minimal treatment such as specific inhaled therapy or spontaneously, whereas others can lead to hospitalization, emergency room visits or fatal evolution (7). Recognition of asthma severity helps the patient to make appropriate changes in physical environment, activity level and medication use in order to implement the self management (16).

\section{Paraclinical investigations}

The gold standard test for measuring objective lung functions is spirometry. Spirometry is used for documentation of the expiratory airway obstruction by measuring: forced vital capacity (FVC), forced expiratory volume in the first second (FEV1) and peak expiratory flow rate (PEF). In adults, taking into account the 2018 GINA criteria for diagnosis, the cutoff for obstruction is defined by a reduced FEV1/FVC ratio of 0.70 or below the lower limit of normal (lowest $5 \%$ of the reference population) and an increase in FEV1 of greater than $12 \%$ and $200 \mathrm{~mL}$, meaning bronchodilator reversibility, after administration of a bronchodilatator (usually 200 to $400 \mu \mathrm{g}$ of $\beta 2$ short-acting agonist). This finding indicates the positive diagnosis of asthma and settles the differential diagnosis with other respiratory conditions (1).

Obstruction may not be present at all times in a patient but its variation should be documented once airway obstruction is confirmed by spirometry. For example a peak-flow meter can be used to do so, a variability of over $10 \%$ in adults or over $13 \%$ in children is indicative of variable expiratory airway obstruction. In order to establish the differential diagnosis, other investigations are required. Allergy documentation include skin prick testing and the level of specific immunoglobulin E in serum, but the results should be interpreted considering the patients' history. In addition, fractional inhaled nitric oxide (FeNO) testing is also used to support the diagnosis of asthma because it is correlated with the levels of blood and sputum eosinophils. In addition, chest radiography may be useful for the differential diagnosis with other respiratory pathologies in the presence of nonspecific symptoms $(1,15)$.

\section{Related comorbidities}

Comorbidities are defined as being coexisting diseases or conditions that influence asthma severity, management or diagnosis and lead to multiple exacerbations and increased hospitalization risk. They are also associated with higher mortality, poor adherence to therapeutic interventions and reduced quality of life $(18,19)$.

Asthma is frequently associated with multiple comorbidities, the most common being: sinusitis, rhinitis, chronic obstructive pulmonary disease (COPD), obesity, obstructive sleep apnea (OSA), gastroesophageal reflux disease, hormonal disorders, diabetes, dermatologic conditions (nettle rush), cardiac disorders (hypertension) and psychopathologies $(18,20)$.

COPD is a comorbidity often confused with asthma and it is characterized by airflow limitation and persistent symptomatology due to small airway diseases and parenchymal destruction (21). The symptoms of COPD and asthma are very similar, but wheezing is more frequent in patients with COPD. Treatment approaches are similar with a few exceptions. Despite the fact that asthma and COPD are viewed by some individuals as totally separate conditions, others consider these disorders as part of a continuum airflow obstruction. The differential diagnosis is important in order to establish the optimum management plan, both pharmacologic and alternative $(18,22)$.

Asthma is often associated with obesity, which by itself is characterized by adipose tissue inflammation and disturbed energy metabolism and it's linked to an increased risk of asthma exacerbations $(23,24,25)$. Overweight individuals are more likely to develop asthma than individuals with normal weight (23). Obesity leads to worse asthma control, reduced lung function, poorer quality of life, poor responses to corticosteroids and important symptoms (23-26). Weight loss strategies improve control of asthma and quality of life. There are many studies investigating the effectiveness of weight loss interventions, such as diet (Mediterranean diet) (27), behavioral therapy, physical activity and bariatric surgery (23). A lot of studies highlighted the association of obesity and OSA in patients with asthma (27).

Obstructive sleep apnea is one of the most frequent comorbidities associated with asthma (24). Both 
asthma and OSA have many nocturnal and diurnal symptoms in common. OSA symptoms are common in asthmatic patients including poor asthma control, daytime sleepiness and reduced quality of life and a higher risk of asthma exacerbations $(24,28)$. OSA is more frequent in patients with severe asthma than in moderate asthma, which may be linked to the potential pathophysiologic interaction between asthma and OSA, both having airway obstruction and systemic inflammation in common. The main type of respiratory events recorded in asthmatic patients are hypopneas rather than apneas (24). Also, patients with severe asthma showed increased apneahypopnea index and poor sleep quality than those with controlled asthma, which requires an individualized treatment plan $(24,29,30)$.

Optimal asthma control requires accurate diagnosis, implementation of effective therapy, and evaluation of coexisting conditions.

\section{Management of asthma patients}

The main goals of asthma management are to achieve good symptom control, minimize the risks of exacerbations, decrease rescue medication intake, improve the quality of life by decreasing respiratory system inflammation and ameliorating the patient's lung function $(31,32)$.

There are many therapies available for asthma management according to Global Initiative for Asthma Guide (1), but in this direction the ability to use the inhaler correctly is a cornerstone. Bad technique is linked to poorer asthma control, multiple exacerbations and increased hospitalization risk $(1,31)$. Despite effective pharmacotherapy, asthma continues to impair quality of life for most patients. Due to the difficulty of controlling symptoms and exacerbations, the need of developing complementary options of treatment is increasing in order to achieve an optimum control and a lower risk of acute episodes or fatal events (33).

Pulmonary rehabilitation is suggested for asthma patients when adequate medical therapy according to GINA 2018 recommendations poorly control the symptoms and mental, physical or social consequences of illness persist during the daily life $(1,2)$, although, it is hardly accepted as an effective treatment for patients with chronic respiratory diseases (3).

However, the available studies proved that the rehabilitation program has shown positive effects regarding clinical symptoms, quality of life, exacerbations, physical functions and healthcare resource utilization (2).

The following non-drug therapy components are included in the rehabilitation program: physical training, comprehensive smoking cessation program, comprehensive patient education, respiratory physiotherapy, psychosocial support, and comprehensive nutritional counseling (2).

Asthmatics usually show a poorer level of physical training by experiencing many respiratory symptoms like chest tightness or breathlessness during exercise leading to an important difficulty in performing daily activities. Unfortunately, the presence of these symptoms may discourage them from pursuing exercise training. Even if Ram et al. demonstrated that physical training showed no improvement in lung function, the quality of life improves in patients who participate in exercise training. This improvement has been related to a reduction in asthma medication intake, higher number of days without symptoms and reduced risk of exacerbations $\mathrm{B}(34)$. Exercise and activities that focus on promoting lung capacity and regulating breathing include yoga, tennis, baseball, using an elliptical machine, biking, walking and swimming are highly recommended for asthmatic patients $(35,36)$. On the other hand, some forms of exercise can cause asthma flare-ups, leading to chest tightness and wheezing. Asthmatic people should avoid high-intensity activities like running, soccer and exercises that involve cold weather such as ice hockey, skiing and winter sports. In conclusion, aerobic training decreases bronchial hyperresponsiveness and serum pro-inflammatory cytokines, improves asthma control and reduces anxiety and depression (36,37).

High altitude (HA) climate (1500-2500m above sea level) is recommended for asthmatic patients because of the decrease of air pollution and small number of allergens such as house dust or storage mites. On the other hand, at HA there is a significant reduction of activated $\mathrm{T}$ lymphocytes, eosinophil cationic protein, eosinophils in blood or sputum and urine leukotrienes (38).

In addition, in the study of Virchow et al it was found an increase in lung function parallel to the decrease of inflammatory markers in patients with intrinsic asthma (39). However, the opinions are contradictory, because Cogo et al. postulated that hypoxia, hyperventilation of cold and dry air and physical 
exertion may worsen asthma or enhance bronchial hyper-responsiveness (40).

As mentioned before, tobacco smoke is a powerful asthma trigger, so smoking cessation is one of the most important strategies in the management plan of asthmatic patients (41). Smoking is a risk factor for asthma, COPD, OSA and lung cancer. Helping patients to quit smoking, improves the prognosis of tobacco-related diseases, the effectiveness of their treatments and the quality of life (42). Smoke can worsen asthma by narrowing the airways, triggering mucus production and damaging the cilia, resulting in the development of emphysema and the retention of secretions (43). In order to obtain smoking cessation, the clinicians should adopt a complex management plan including pharmacotherapy and behavioral support. Pharmacological treatment is based on three approved medications: nicotine replacement therapy, bupropion and varenicline which should be integrated beside behavioral support in the patients' daily life. Nicotine replacement therapy include different forms of substitution such as patch, tablet, gum and oral spray, delivering nicotine more quickly, but effects are short-lived. Bupropion is the most widely use antidepressant, Which acts as an antagonist of reabsorption of noradrenaline and dopamine. Varenicline is a partial agonist for the $\alpha 4 \beta 2$ nicotinic acetylcholine receptor subtype (nACh) that has the capacity to reduce the feelings of craving and withdrawal caused by smoking cessation. Medication is chosen considering patient preference, past experience, cost, contraindications and efficacy. The most efficacious pharmacotherapy treatments are combination nicotine replacement therapy or varenicline. Cahill et al. proved that nicotine replacement therapy is more effective than placebo or nicotine replacement monotherapy, but nicotine replacement therapy in conjunction with bupropion has a more significant effect (44). Chang et al showed that varenicline in addition to a nicotine replacement patch is more efficacious than varenicline alone, but, however, combined treatment with varenicline and bupropion increases short-term but not long-term cessation rates versus treatment with varenicline alone $(45,46)$.

Counselling and intensive cessation advices are more helpful in making smokers to quit compared with brief advice. Although, as Hughes et al proved they can be supplemented with self-help materials and referrals to individual, telephone quit lines, internet- based interventions and group-based counselling programs. Counselling typically concentrate on problem-solving skills, coping techniques, relaxation training, group counselling adding elements of peer support $(13,47)$.

Enviromental control practices are integral components of asthma management, which have been shown to reduce asthma exacerbations and symptoms. An environmental history needs to be obtained in order to evaluate the environmental exposures. This history includes questions regarding the presence of indoor and outdoor pollutants that can trigger asthma symptoms. Environmental control strategies include source control, source removal, and mitigation strategies, such as allergen proof mattress, pillow encasements and high-efficiency particulate air purifiers (10).

While drug treatment can control the symptoms of asthma in the majority of patients, alternative interventions (inspiratory muscle training, breathing techniques, manual therapy, speleotherapy, halotherapy) would constitute significant advances in asthma management because they are at low risk, effective and have long-term benefits (48).

Inspiratory muscle training (IMT) is a technique used to increase the endurance and strength of diaphragm and accessory muscles of respiration process. Different forms of IMT have been used such as flow resistive loading, pressure threshold loading and normocapnic hyperpneea (NH) (49).

Flow restrictive loading ( $F R L$ ) consists in breathing through a device with a variable diameter orifice which increases the inspiratory pressure required to generate a given airflow. This tool acts like a pressure manometer that has a $2 \mathrm{~mm}$ leak and sends a link to a computer containing the software necessary to run the "Test of Incremental Respiratory Endurance" (TIRE). This leak is required in order to set a resistance to the inspiratory flow. Following the TIRE protocol, each subject is first asked to forcefully exhale and go to residual lung volume (RV) - expiration unloaded, and then immediately inspire as much as they can, against the $2 \mathrm{~mm}$ leak resistance, going from RV to total lung capacity until the end of the task. A computer then recorded the effort during the test as sustained maximum inspiratory pressure (SMIP), shown as the area below the curve. The study of Shei et al proved that the programmes are recommended 3 days/week for a 6 weeks period (49). As Mickleborough et al showed, the effects of FRL are benefic on asthma 
subject, improving the breathing mechanisms, the oxygen cost, the ventilation and the endurance exercise. Also, this method has a good influence on the symptoms, reducing the sensation of fatigue, improving quality of life and decreasing the use of $\beta 2$ agonist $(49,50)$.

Pressure threshold loading consists in a method which requires a patient to breathe through a device with a one-way valve that remains closed until a predetermined amount of inspiratory generated pressure, thereupon the valve opens and airflow is allowed. This method leads to a dislocation between intra-airway pressure and pleural pressure, increasing airway stretch. It was observed an improvement in FEV1 and peak expiratory flow rate post-IMT consisting in pressure threshold loading. In addition, short-acting 32 agonist usage has been shown to reduce, suggesting an improvement in the severity of disease per se. So, pressure threshold loading may provide a unique stretching stimulus to the airway and deep inspiration in people in whom asthma is not causing a Broncho constrictor response (51).

The role of normocapnic hyperpneea (NH), which is one of respiratory muscle endurance training (RMET), is to maintain a high level of ventilation per minute, correlating to the maximum voluntary ventilation of the patients, carbon dioxide levels being stabilized with rebreathing. Taking into account that the equipment for maintaining $\mathrm{CO} 2$-homeostasis during hyperpnoea is both complicated and expensive, $\mathrm{NH}$ has not been used on a large scale. A solution to preserve $\mathrm{CO} 2$-homeostasis would be to enlarge the dead space of the ventilatory system, which could be achieved by breathing through a tube. This could prove to be a safe and convenient solution, thus enabling its widespread use as a inexpensive and new method of RMET $(49,52)$. Bernardi et al reported that $\mathrm{NH}$ could be the most appropriate technique of improving respiratory muscle endurance (53). $\mathrm{NH}$ has positive effects on asthma symptoms. One of the mechanisms consists in repetitive airway stretching with increased ventilation during exercise to see that intense deep breathing would beneficially affect airway inflammation and bronchial hyperreactivity. Philipp et al proved that the repetitive hyperpnea is used as a non pharmacological tool to ameliorate bronchial reactivity, sensitivity and airway inflammation (54).

Finally, IMT may be beneficial for reducing both exertional and resting dyspnea, as well as reducing the use of medication. These changes have been linked to increases in inspiratory muscle strength and improving exercise capacity (49).

\section{Breathing techniques (BT)}

Breathing exercises often form part of a complex of techniques involving respiratory and non-respiratory components.

Respiratory Breathing exercises are represented by exercises that encourage patients to breathe through the nose to reduce thoracic movement by using shallow breathing. Patients are advised to practice the techniques regularly and to incorporate them into their daily routine.

There are three main groups of breathing exercises:

- techniques used to lower hyperventilation, like Papworth method and the Buteyko breathing exercise, non-hyperventilatory techniques such as yoga breathing and techniques for improving breath control like playing musical instruments or singing - exercises aimed to improve endurance or strength of the respiratory muscles and diaphragm like inspiratory muscle training

- techniques that enhance the flexibility of the thoracic cage and improving posture such as musculoskeletal training.

Freitas et al showed that the subjects performing BT achieved a statistically significant reduction in medication use and self-reported symptoms, but with no improvement in pulmonary function $(55,56)$. The Alexander Technique (AT) involves a set of movements meant to correct the posture and reset the body's natural alignment and is used particularly for asthmatics patients in order to ease their efforts of breathing $(48,55)$.

The most useful non respiratory components of breathing techniques seen in clinical practice are yoga training and manual therapy. Yoga training can involve breathing exercises, physical postures and meditation and is usually accompanied by such as music therapy which can involve listening to music, singing, playing an instrument and dancing. These therapies show a decrease in symptoms but the methodological limitations of the studies allowed only weak recommendations in asthma rehabilitation. Manual therapy is represented by chiropractic and osteopathic techniques, involving a multidisciplinary team including physiotherapists, respiratory therapists, chiropractic and osteopathic physicians in order to improve the mobility of the ribcage and the spine spine to increase lung function (55). However, 
there is insufficient evidence involving this therapy in asthma management, so, more research is needed (57). Also, as Brinkhaus et al showed acupuncture is a cost-effective tool used as adjunct to the routine care of allergic asthma, which improves both specific symptoms and general quality of life (58).

Speleotherapy or salt therapy consists in the inhalation of small salt particles from natural salt caves and is a treatment method for people with chronic and allergic respiratory diseases $(59,60,61)$. Speleotherapy is widely used in COPD patients, but in asthma there is no clear evidence of its efficacy. Also it can be considered as an helpful treatment, due to air quality, underground, climate, irradiation, and hyperosmolar environment. In addition, for the patients' safety, monitoring of FeNO levels pre- and post speleotherapy can be used to track the level of allergic inflammation (62).

Halotherapy it is often used as an alternative for speleotherapy. It is a drug-free therapeutic method and consists in sitting in a room coated with salt crystals, designed to mime some benefits of the microclimate of natural salt caves (62). Benefits are believed to come from underground climate, air quality, radiation and air pressure (63). Effects of halotherapy climate are plenty, such as bactericidal effect, immunological changes and improvement of immunity, but the mechanisms by which it is beneficial remain unclear $(62,63)$.

\section{Conclusions}

Asthma is a heterogeneous condition that remains poorly controlled in many cases despite optimum pharmacological management according to GINA 2018 guidelines. The additional interventions in asthma management, such as physical training, exposure to high altitude, smoking cessation, environmental control practices, inspiratory muscle training, breathing techniques, speleotherapy and halotherapy have been proven to improve muscle strength, exercise capacity and symptomatology. Also, they have been associated to fewer exacerbations and a lower use of rescue medication, leading to a better quality of life.

\section{References}

1. Pedersen S, Reddel H, Boulet LP. Global Initiative for Asthma. Global Strategy for Asthma Management and Prevention, 2018. Available at https://ginasthma.org/2018-gina/

2. Schultz K, Seidl H, Jelusic D, Wagner R, Wittmann M, et al. Effectiveness of pulmonary rehabilitation for patients with asthma: study protocol of a randomized controlled trial (EPRA). BMC Pulmonary Medicine. 2017;17(1):49.

3. Lingner $\mathrm{H}$, Ernst $\mathrm{S}$, Großhennig $\mathrm{A}$, Djahangiri $\mathrm{N}$, Scheub D et al. Asthma control and health-related quality of life one year after inpatient pulmonary rehabilitation: the ProKAR Study. Journal of asthma. 2015; 52:614-21.

4. Lundbäck B, Backman H, Lötvall J, Rönmark E. Is asthma prevalence still increasing? Expert Review of Respiratory Medicine.2015;10:39-51.

5. Carson KV, Chandratilleke MG, Picot J, BrinnMP, Esterman AJ, Smith BJ. Physical training for asthma. Cochrane Database of Systematic Reviews 2013, Issue 9. Art. No.: CD001116.

6. Sullivan A, Hunt E, MacSharry J, Murphy D. M. The Microbiome and the Pathophysiology of Asthma. Respiratory Research.2016; 17:163.

7. Mims JW. Asthma: definitions and pathophysiology. International Forum of Allergy \& Rhinology.2015; 5(S1):S2-S6.

8. Felman A. "What is asthma?" Medical News Today. Nov. 2018. Available at: https://www.medicalnewstoday.com/articles/323523.p $\mathrm{hp}$

9. Todea DA, Suatean I, Coman AC, Rosca LE. The Effect of Climate Change and Air Pollution on Allergenic Potential of Pollens Notulae Botanicae Horti Agrobotanici.2013; 41(2):646-50.

10. Matsui EC, Abramson SL, Sandel MT. Indoor Environmental Control Practices and Asthma Management. Pediatrics.2016; 138:25-89.

11. Todea D, Cosma C, Dicu T, Roşca L, Cucoş(Dinu) A, Rişteiu M, Iancu D, Papuc I, Rădulescu D. Lung cancer risk induced by residential radon in CLUJ and Alba Counties, ROMANIA. Environmental Engineering and Management Journal. 2013.12(6):1281-5.

12. D'Amato G, Holgate ST, Pawankar R, Ledford DK, Cecchi L. Meteorological conditions, climate change, new emerging factors, and asthma and related allergic disorders. A statement of the World Allergy Organization. World Allergy Organization Journal.2015;8(1):1-52.

13. Vremaroiu-Coman A, Alexescu TG, Negrean V, Milaciu MV, Buzoianu AD, Ciumarnean L, Todea DA. Ethical aspects of smoking cessation among the population from Transylvania. Balneo Research Journal.2018;9(3):254-9.

14. Budin CE, Marginean C, Bordea IR, Enache LS, Enache EL, Grigorescu BL, Biro L, Rusu E, Nemes RM, Todea DA. The Influence of Smoking on Nicotine Exposure Biomarkers and Inflammatory Profile Among Foster Care Teenagers, Romania. Rev Chim. 2018;69(12):3659-63. 
15. Brigham, E. P., \& West, N. E. Diagnosis of asthma: diagnostic testing. International Forum of Allergy \& Rhinology.2015; 5(S1), S27-S30.

16. Ciprandi G, Schiavetti I, Sorbello V, Ricciardolo FL. Perception of Asthma Symptoms as Assessed on the Visual Analog Scale in Subjects With Asthma: A Real-Life Study. Respiratory Care. 2015; 61(1):23-29

17. Fuhlbrigge A, Peden D, Apter A J, Boushey HA, Camargo CA, Gern J ,Blaisdell C. Asthma outcomes: Exacerbations. Journal of Allergy and Clinical Immunology.2012; 129(3):S34-S48

18. Ledford DK, Lockey RF. Asthma and comorbidities. Current Opinion in Allergy and Clinical Immunology.2013; 13(1): 78-86.

19. Su X, Ren Y, Li M, Zhao X, Kong L, Kang J. Prevalence of Comorbidities in Asthma and Nonasthma Patients. Medicine.2016; 95(22), 34-59.

20. Boulet LP, Boulay MÈ. Asthma-related comorbidities. Expert Review of Respiratory Medicine.2011; 5(3):377-93.

21. Global Initiative for Chronic Obstructive Lung Disease. Global Strategy for the Diagnosis, Management, and Prevention of Chronic Obstructive Pulmonary Disease: 2019. https://goldcopd.org/wpcontent/uploads/2018/11/GOL D-2019-v1.7-FINAL14Nov2018 WMS.pdf. Accessed February 7, 2019

22. 22.Alexescu TG, Maierean A, Ciumarnean L, Budin C, Dogaru G, Todea DA. Rehabilitation therapies in stable chronic obstructive pulmonary disease. Balneo Research Journal. 2019; 10(1):37-44

23. Candemir I, Ergun P, Kaymaz D. Efficacy of a multidisciplinary pulmonary rehabilitation outpatient program on exacerbations in overweight and obese patients with asthma. Wiener Klinische Wochenschrift.2017; 129(19-20):655-64.

24. Bonsignore MR, Baiamonte P, Mazzuca E, Castrogiovanni A, Marrone O. Obstructive sleep apnea and comorbidities: a dangerous liaison. Multidisciplinary Respiratory Medicine.2019; 14(1):8.

25. Rusu A, Todea D, Rosca L, Nita C, Bala C. The development of a sleep apnea screening program in Romania type 2 diabetic patients: a pilot study. Acta Diabetologica.2012.49(2):105-9.

26. Farah CS, Kermode JA, Downie SR., Brown NJ., Hardaker KM., Berend N., Salome C. M. Obesity Is a Determinant of Asthma Control Independent of Inflammation and Lung Mechanics. Chest.2011; 140(3): 659-666

27. Todea D, Cadar O, Simedru D, Roman C, Tanaselia C, Suatean I, Naghiu A, Determination of Major-to-Trace Minerals and Polyphenols in Different Apple Cultivars. Not Bot Horti Agrobo. 2014;42(2):523-529.

28. Rusu A, Nita C, Todea D, Rosca L, Bala C, Hancu N. Correlation of the daytime sleepiness with respiratory sleep parameters in patients with sleep apnea and type 2 diabetes. Acta Endocrinologica. 2011;VII(2):163-71.

29. Radescu OD, Albu S, Baciut MS, Coman AC, Bechir ES, Pacurar M, Todea DA. Results in the Treatment with Twin Block Polymeric Appliance of the Retrognathic Mandible in Sleep Apnea Patients. Materiale Plastice.2017;54(3):473-476.

30. Coman AC, Todea DA, Popa E, Radu T, Cadar O, Borzan C. Multilateral characterization of masks and tubes surfaces in contact with respiratory system through ventilation, Journal of Optoelectronics and Advanced Materials. 2015;17(9-10):1563-1571.

31. Yawn BP, Han MK. Practical Considerations for the Diagnosis and Management of Asthma in Older Adults. Mayo Clinic Proceedings. 2017; 92(11):1697-1705

32. Farid R, Azad FJ, Atri AE, Rahimi MB, Khaledan A, Talaei-Khoei M, Ghafari J, Ghasemi R . Effect of aerobic exercise training on pulmonary function and tolerance of activity in asthmatic patients. Iran J Allergy Asthma Immunol. 2005;4(3):133-8.

33. Bruton A, Lee A, Yardley L, Raftery J, Arden-Close E, Kirby S, Thomas M. Physiotherapy breathing retraining for asthma: a randomised controlled trial. The Lancet Respiratory Medicine.2018; 6(1):19-28.

34. Ram FSF, Robinson SM, Black PN. Physical training for asthma. The Cochrane Database of Systematic Reviews 2000, Issue 1. Art. No.: CD001116. Available from:

https://www.ncbi.nlm.nih.gov/pubmed/16235280

35. Fletcher J. What are the best types of exercise for asthma?, Medical News Today. 2019. Available at: https://www.medicalnewstoday.com/articles/324445.p hp

36. França-Pinto A, Mendes FA, de Carvalho-Pinto $\mathrm{RM}$, Agondi RC, Cukier A, et al. Aerobic training decreases bronchial hyperresponsiveness and systemic inflammation in patients with moderate or severe asthma: a randomised controlled trial. Thorax. 2015;70(8):732-9.

37. Brennan D, Asthma and exercise. 2019. Available at: https://www.webmd.com/asthma/guide/exercisingasthma? fbclid=IwAR0yq7dh3Holsclg9J7wFYCzYJp b24vo3pVdkg3QdhXE_CpgYIf1E2n--N4\#2

38. Menz G. Effect of sustained high altitude on asthma patients. Expert Review of Respiratory Medicine.2007; $1(2): 219-25$.

39. Virchow JC, Oehling A, Boer L et al. Pulmonary function, activated $\mathrm{T}$ cells, peripheral blood eosinophilia, and serum activity for eosinophil survival in vitro: a longitudinal study in bronchial asthma, J. Allergy Clin. Immunol. 1994; 94:240-49.

40. Cogo A, Basnyat B, Legnani D, Allegra L. Bronchial Asthma and Airway Hyperresponsiveness at High Altitude. Respiration. 1997; 64(6):444-49. 
41. Chaudhuri R, Livingston E, McMahon AD, Lafferty $\mathrm{J}$, Fraser I, et al. Effects of Smoking Cessation on Lung Function and Airway Inflammation in Smokers with Asthma. Am J Respir Crit Care Med.2006. Vol 174(2):127-133.

42. Perriot J, Underner M, Peiffer G, Dautzenberg B. L'aide à l'arrêt du tabac des fumeurs atteints de BPCO, d'asthme, de cancer bronchique, et opérés. Revue de Pneumologie Clinique. 2018; 74(3): 170 180.

43. Burgess L. How does smoke affect people with asthma? Medical News Today. 2019 Available at https://www.medicalnewstoday.com/articles/324444.p hp

44. Cahill K, Stevens S, Perera R, et al. Pharmacological interventions for smoking cessation: an overview and network metaanalysis. Cochrane Database Syst Rev 2013;(5):CD009329.

45. Chang $\mathrm{PH}$, Chiang $\mathrm{CH}$, Ho WC, et al. Combination therapy of varenicline with nicotine replacement therapy is better than varenicline alone: a systematic review and meta-analysis of randomized controlled trials. BMC Public Health. 2015;15:689.

46. Ebbert JO, Hatsukami DK, Croghan IT, et al. Combination varenicline and bupropion SR for tobacco-dependence treatment in cigarette smokers: a randomized trial. JAMA 2014;311:155-63.

47. Hughes JR. An updated algorithm for choosing among smoking cessation treatments. J Subst Abuse Treat .2013;45:215-21.

48. Dennis JA, Cates CJ. Alexander technique for chronic asthma. Cochrane Database of Systematic Reviews 2000, Issue 2. Art. No.: CD000995

49. Shei RJ, Paris HLR, Wilhite DP, Chapman RF, Mickleborough TD. The role of inspiratory muscle training in the management of asthma and exerciseinduced bronchoconstriction. The Physician and Sportsmedicine. 2016; 44(4):327-34.

50. Mickleborough TD, Nichols T, Lindley MR, Chatham $\mathrm{K}$, Ionescu AA. Inspiratory flow resistive loading improves respiratory muscle function and endurance capacity in recreational runners. Scandinavian Journal of Medicine \& Science in Sports.2009; 20(3):458-68.

51. How SC, Romer LM, McConnell AK. Acute effects of inspiratory pressure threshold loading upon airway resistance in people with asthma. Respiratory Physiology \& Neurobiology.2009; 166(3):159-63.

52. Koppers RJH, Vos PJE, Folgering HTM. Tube breathing as a new potential method to perform respiratory muscle training: Safety in healthy volunteers. Respiratory Medicine.2006; 100(4):71420.

53. Bernardi E, Pomidori L, Bassal F, Contoli M, Cogo A. Respiratory muscle training with normocapnic hyperpnea improves ventilatory pattern and thoracoabdominal coordination, and reduces oxygen desaturation during endurance exercise testing in COPD patients. International Journal of Chronic Obstructive Pulmonary Disease. 2015; Int J Chron Obstruct Pulmon Dis. 2015 Sep 10;10:1899-906.

54. Philipp A. Eichenberger, Kurzen A, Stephanie N. Diener, Thomas A. Scherer, Christina M. Spengler. Repetitive, intense hyperpnea to reduce bronchial reactivity in asthmatics - A pilot studyEuropean Respiratory Journal .2016; 48(60). Available at: https://erj.ersjournals.com/content/48/suppl_60/PA22 73

55. Bruton A. Breathing exercises for adults with asthma. Drug and Therapeutics Bulletin. 2015; 53(11):126-29.

56. Freitas DA, Holloway EA, Bruno SS, Chaves GS, Fregonezi GA, Mendonça KM. Breathing exercises for adults with asthma. Cochrane Database of Systematic Reviews.2013; issue 10.

57. Marshall $M$, Rathbone J. Early Intervention for psychosis. Cochrane Database of Systematic Reviews 2006, Issue 4. Art. No.: CD004718

58. Brinkhaus B, Roll S, Jena S, Icke K, Adam D, Binting S, Witt C. M. Acupuncture in Patients with Allergic Asthma: A Randomized Pragmatic Trial. The Journal of Alternative and Complementary Medicine. 2017; 23(4):268-77.

59. Todea D, Herescu A, Rosca L. Obstructive Sleep Apnea Syndrome - a matter of public health, Transylvanian Review of Administrative Sciences. 2012; 37E:186-201.

60. Danteș E., Lilios G., Fildan A.P. Environmental tobacco smoke exposure - an independent risk factor for lung cancer? Journal of Environmental Protection and Ecology (JEPE), 2014, vol. 16/issue 4, pp 16201626.

61. Tudorache E, Fildan AP, Frandes M, Dantes E, Tofolean D. Aging and extrapulmonary effects of chronic obstructive pulmonary disease. Clinical Interventions in Aging (Clin Interv Aging). 2017;12:1281-1287

62. Bar-Yoseph R, Kugelman N, Livnat G, Gur M, Hakim F, Nir V, Bentur L. Halotherapy as asthma treatment in children: A randomized, controlled, prospective pilot study. Pediatric Pulmonology. 2016; 52(5):580-87.

63. MUNTEANU Constantin - SPELEOTHERAPY scientific relevance in the last five years $(2013-2017)$ - A systematic review, Balneo Research Journal. 2017;8(4):252-254, DOI 10.12680/balneo.2017.161. 